\title{
ESTUDO ECOLÓGICO E ZOOGEOGRÁFICO SOBRE A FAUNA DE LAGARTOS (SAURIA) DAS DUNAS DE NATAL, RIO GRANDE DO NORTE E DA RESTINGA DE PONTA DE CAMPINA, CABEDELO, PARAÍBA, BRASIL ${ }^{1}$
}

\author{
Eliza Maria Xavier Freire ${ }^{2}$
}

\begin{abstract}
ECOLOGIC AND ZOOGEOGRAPHIC STUDY ON A FAUNA OF LIZARDS (SAURIA) from Dunas of Natal, Rio Grande do Norte and from Restinga de Ponta de CAMPINA, CABEDELO, PARAíBA, BRAZIL. An inventory of the fauna of lizards was made in the dunes of Natal (Rio Grande do Norte, Brazil) and in the "restingas" (sandbanks with its associated vegetation) of Ponta de Campina (Cabedelo, Paraiba, Brazil) from October/84 to March/86. The objective was to know the composition and structure of these communities as well as to evaluate the zoogeographical importance of the spots. The samples were collected and the observations were made along of transects which were established to enable the sampling of the habitats under study in both areas. Each transect was crossed one day per month, during 14 months. The collect of lizards was made manually or through the use of a small gun, calibre 22, with ammunition of the type mustard lead. Observations were made on the habitats utilized by the species (open areas or forests) and on the species distribution by microhabitat. The fauna of lizards in the area under study is formed by species ecologically connected with open formations and also by species of the Atlantic Rain Forest. The majority of the species collected in the open areas is part of the fauna of the great diagonal of open formations which come from the Northeast of Argentina and go up to the state of Maranhão. There is, however, a great influence of the caatinga fauna, specially in the dunes of Natal. The species found in the forest of the dunes, in spite of being part of the Atlantic Forest, are morphologically differentiated. Gymnodactylus darwinii (Gray, 1845) and G. geckoides Spix, 1825 are sympatrics, but not syntopics, in the dunes of Natal.

KEY WORDS. Sauria, lizards, ecology, restinga, Brazil
\end{abstract}

As dunas e restingas da costa brasileira são formações arenosas recentes edificadas diretamente ou a partir de depósitos marinhos retrabalhados. As sucessivas flutuações do nível do mar no Quaternário são responsáveis pela configuração e distribuição da maior parte deste tipo de ecossistema na costa atual (SouZA 1945; LACERDA et al. 1982; HALLEGOUET 1984). As dunas e restingas não têm, certamente, a expressão de domínio morfoclimático, entretanto, recebem a influência florística de alguns desses domínios, como o da Mata Atlântica, ao longo de praticamente toda a costa leste e, no seu extremo norte, o das Caatingas.

As primeiras referências sobre restingas datam dos séculos XVI e XVII e incluem descrições dos naturalistas sobre a flora, a fauna e as paisagens naturais do

1) Trabalho desenvolvido com auxilio da CAPES.

2) Departamento de Zoologia, Museu de História Natural Universidade Federal de Alagoas. Rua Aristeu de Andrade 452, 57021-090 Maceió, Alagoas, Brasil. 
Brasil. Somente após 1900, podem ser destacados estudos sistemáticos, ecológicos e zoogeográficos, como o de NovaEs (1950), sobre a distribuição das aves em Sernambetiba, o de CASTRO (1952), sobre a presença do gênero Tylos (Isópoda) nessa mesma restinga e o de PEIXоTo (1977), sobre os anfibios associados a bromeliáceas nas restingas do Rio de Janeiro e Espírito Santo. Os trabalhos mais recentes (HAY et al. 1981; VALLEJo \& VALLEJo 1981; ARAÚJO \& HENRIQUES 1984; MACIEL 1984; OTERO 1984; PORTO \& TEIXEIRA 1984; ARAÚJO 1985; ROCHA 1988, 1990, 1993) caracterizam-se por apresentar uma maior preocupação com a ecologia das diversas restingas brasileiras, com suas comunidades animais e sua preservação. PORTO e TEIXEIRA (1984), comparando as avifaunas das restingas de Maricá, Rio de Janeiro e Valença, Bahia, sugerem que estas funcionem como "tampão" para as espécies da Mata Atlântica. Também destacam sua importância para a conservação, uma vez que a destruição da mata tornaria as restingas refúgios para parte de sua fauna.

Apesar do recente interesse pelo estudo das restingas, os trabalhos sobre suas comunidades animais ainda são relativamente escassos, conforme apontado por LACERDA et al. (1982). No que diz respeito à herpetofauna, essa escassez é ainda mais evidente, destacando-se apenas os estudos de ARAúJo (1985) e ROCHA (1988, 1990, 1993) sobre os lagartos da restinga de Maricá, Rio de Janeiro.

No Nordeste, a situação é mais grave, pois a inexistência de estudos sobre as restingas se soma ao crescente e destruidor processo de ocupação humana nesses ecossitemas. As dunas de Natal (Rio Grande do Norte) e a restinga de Ponta de Campina (Cabedelo, Paraíba) têm sofrido constantes processos de destruição devido, principalmente, à especulação imobiliária. Tais destruições têm ocorrido antes que qualquer estudo faunístico tenha sido realizado. $\mathrm{O}$ estudo comparativo entre a fauna de lagartos dessas duas restingas, que aqui se apresenta, além de auxiliar no conhecimento sobre a estrutura das comunidades de lagartos de diferentes restingas, permite discutir a importância zoogeográfica destas áreas.

\section{ÁREA DE ESTUdo}

\section{Dunas de Natal}

As dunas estudadas localizam-se na faixa costeira da região oriental de Natal entre $5^{\circ} 47^{\prime} 25^{\prime \prime}$ e $5^{\circ} 51^{\prime} 56^{\prime \prime S}$ e $35^{\circ} 10^{\prime} 47^{\prime \prime}$ e $35^{\circ} 11^{\prime} 59^{\prime \prime W}$. Compreendem $9 \mathrm{~km}$ de extensão, entre o Farol de Mãe Luíza e o marco inicial da rodovia RN-063, que liga a cidade à praia de Ponta Negra (SECPLAN/RN 1981) (Fig. 1). Elas formam cordões alongados e paralelos à costa, dispostos no eixo SE-NW, que corresponde à direção dos ventos alíseos atuais (NoGUEIRA 1982). Nesta área, uma transecção estabelecida no sentido mar-continente, permite reconhecer quatro regiões fisionomicamente distintas e grosseiramente paralelas à linha de costa: as dunas costeiras, as dunas fixas, vales entre-dunas e o tabuleiro.

As dunas costeiras (ou área supra-litorânea) são móveis, sem cobertura vegetal ou com vegetação herbácea rala fixadora de areia. Eventualmente, essas dunas supra-litorâneas podem estar recobertas por uma vegetação arbórea-arbustiva, formando moitas esparsas. 


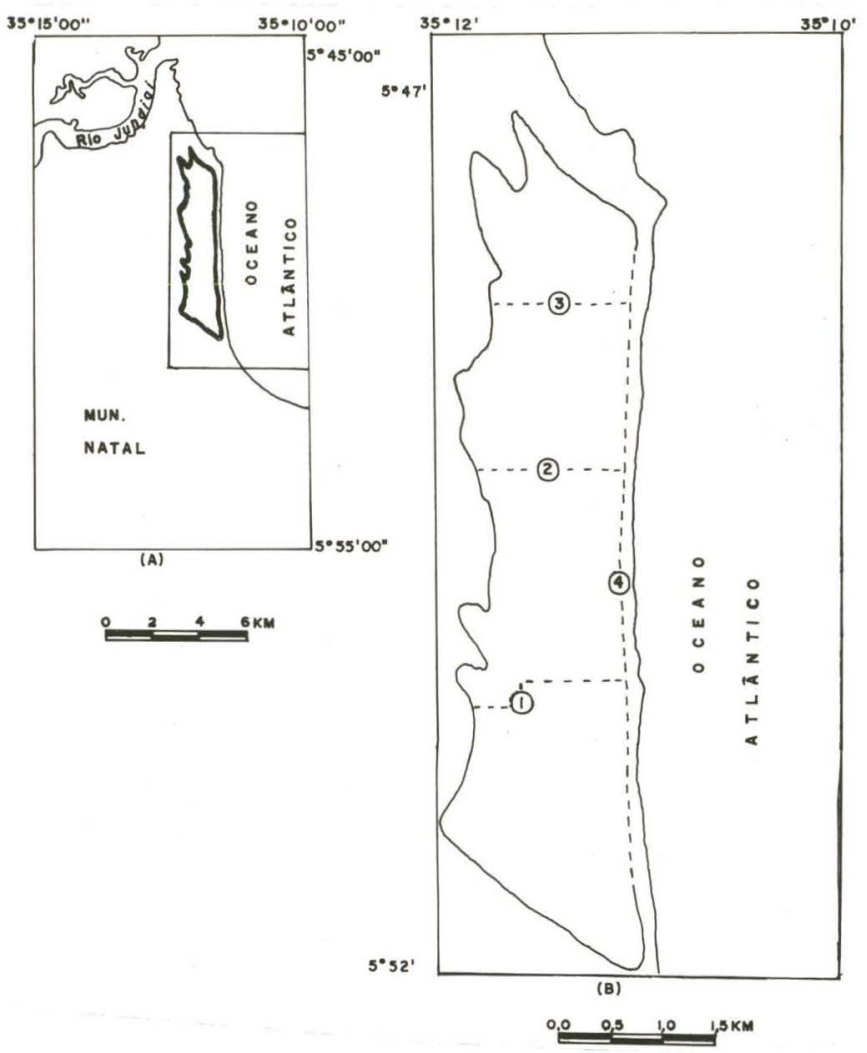

Fig. 1. Localização das dunas costeiras de Natal, Rio Grande do Norte. (A) Município de Natal com a localização da área de estudo; (B) área de estudo ampliada destacando as transecções (números 1 a 4).

Mais para o interior do continente, encontram-se dunas fixas, muito maiores, algumas delas atingindo cerca de 90 metros acima do nível do mar (TAVARES 1960; RIZZINI 1979). A vegetação é predominantemente arbóreo-arbustiva, com árvores de três a sete metros de altura, especialmente marcada pela presença do cajueiro (Anacardium occidentale Linn.). Em alguns locais, geralmente no topo de algumas dunas, observam-se enormes clareiras com moitas arbustivas esparsas, sob as quais encontram-se grandes touceiras da bromeliácea Hohembergia ramageana Mez.

Intercalando esses cordões de dunas fixas, encontram-se vales com até 30 metros de profundidade (TAVARES 1960), revestidos por uma floresta com árvores que atingem 15 ou mais metros e com estrato herbáceo pouco desenvolvido. Esta terceira região fisionômica corresponde à Floresta Perenifolia Higrófila Costeira de KuHLMANN (1977). Segundo TAVARES (1960), essa mata devia existir antes das dunas e, à medida que estas foram sendo formadas, soterraram-na parcialmente.

A quarta região fisionômica corresponde ao tabuleiro. Este tem início logo após a faixa de dunas fixas e, floristicamente, é semelhante aos cerrados do Brasil 
central (TAVARES 1960; RIZZINI 1979; SECPLAN/RN 1981). O contraste fisionômico existente entre essas quatro regiões não encontra, aparentemente, paralelo nas condições climáticas vigentes. A umidade relativa do ar mantem-se em torno de $80 \%$ ao longo do ano e as precipitações variam entre 1.000 e $1.500 \mathrm{~mm}$ anuais. O trimestre abril-junho é o mais chuvoso, a estação seca vai de outubro a janeiro (SECPLAN/RN 1981; NogUEIRA 1982). Não existem variações locais, toda a área está sujeita ao mesmo regime pluviométrico. As principais causas para explicar a diversidade florística da área devem ser buscadas nos tipos de solo, na influência do mar e, principalmente, na história florística da região. É provável que os contornos destas paisagens, no auge do último período seco, diferiram muito dos atuais.

\section{Restinga de Ponta de Campina}

A restinga de Cabedelo foi edificada durante as flutuações do nível marinho no holoceno (HALLEGOUET 1984). A área estudada, remanescente dessa restinga, compreende aproximadamente $1.000 \mathrm{~m}$ de largura por $900 \mathrm{~m}$ de extensão e está situada na praia de Ponta de Campina, entre 701'29"S e 3450'04"W (Fig. 2). Entre 1984 e 1986, época em que este estudo foi realizado, era uma das poucas áreas naturais que ainda permaneciam na região. Recentemente, ela foi completamente devastada pela especulação imobiliária com a ampliação da BR-230.

Duas formações vegetais caracterizavam a área estudada: a restinga arbustiva e a restinga arbórea ou Floresta Baixa Litorânea Esclerófila (TAVARES DE MELO 1983).

Na restinga arbórea, de modo similar ao que foi observado nas dunas fixas de Natal, o cajueiro (Anacardium occidentale Linn.) era a árvore mais comumente encontrada. A restinga arbórea era constituída por árvores que geralmente não ultrapassavam 15 metros de altura, formando um só estrato.

À altura do Km 11 da BR-230, margem esquerda no sentido João Pessoa Cabedelo, entre $7^{\circ} 03^{\prime} 43^{\prime \prime S}$ e $34^{\circ} 51^{\prime} 09^{\prime \prime}$, situava-se a Mata do AMÉm (Fig. 2), que também foi estudada, tendo em vista que esta deve ter sido contínua com a restinga. A presença de espécies típicas da Mata Atlântica, o porte das árvores (mais de 15 metros) e a sua densidade distinguiam-na da mata esclerófila da restinga. Provavelmente, estendia-se tanto para o norte, como para o sul, até próximo ao estuário do Rio Paraíba. Correspondia à Floresta Estacional Perenifólia Costeira (ANDRADELIMA 1961) ou Floresta Perenifólia Higrófila Costeira (KUHLMANN 1977).

O clima da região é do tipo tropical úmido com três meses secos (outubro a dezembro) e com precipitações variando entre 1.500 e $2.000 \mathrm{~mm}$ anuais (TAVARES DE MELO 1983).

\section{MATERIAL E MÉTODOS}

Os levantamentos foram efetuados através de coletas mensais nas dunas de Natal e na restinga de Ponta de Campina, de outubro de 1984 a novembro de 1985. Devido à falta de coletas nos meses de janeiro a março de 1985 , essas foram realizadas nos mesmos meses de 1986. 


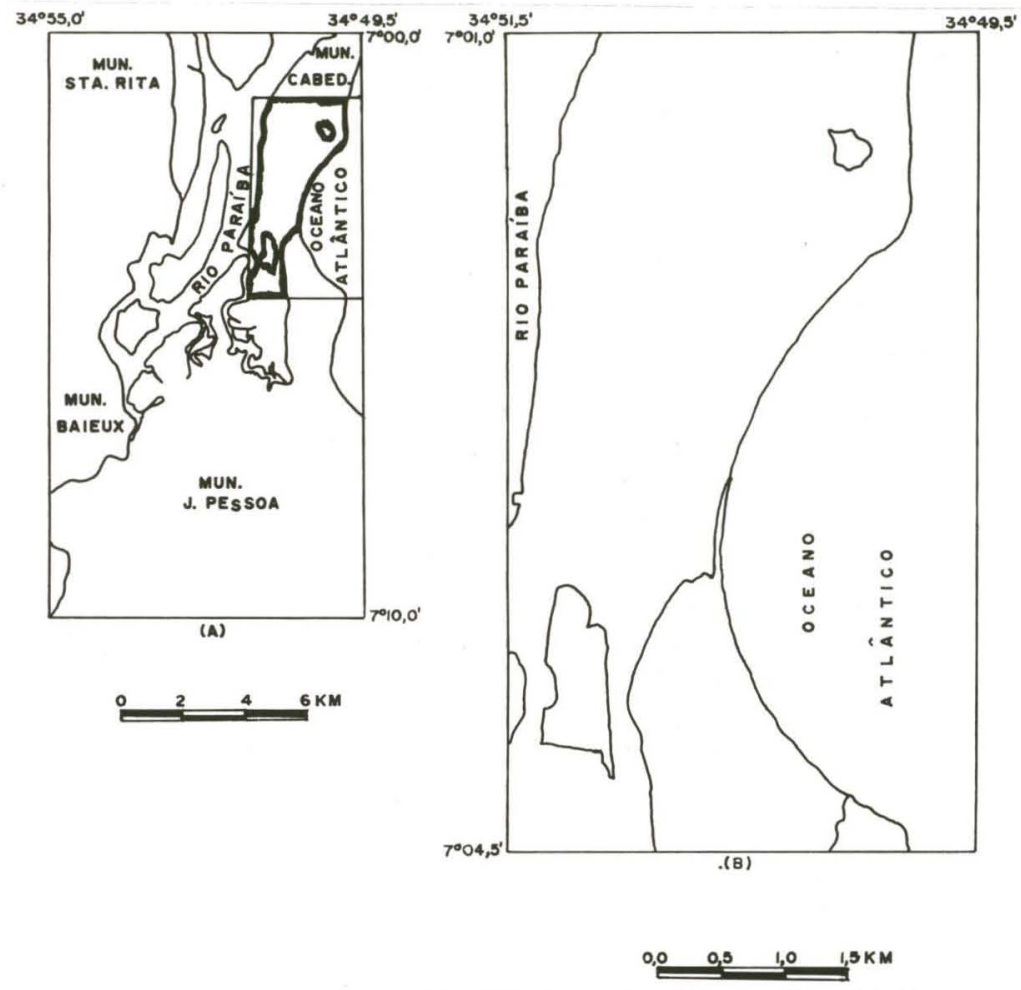

Fig. 2. Localização das áreas estudadas no município de Cabedelo, Paraíba. (A) Municipios de João Pessoa e Cabedelo, com a localização da Restinga de Ponta de Campina (área menor, ao norte) e da Mata do Amém (maior e ao sul); (B) áreas ampliadas.

Para a coleta utilizou-se o método das transecções, que permite uma amostragem dos diversos habitats das dunas e da restinga. Nas dunas de Natal, foram delimitadas quatro transecções: três no sentido continente-mar e uma ao longo da costa (Fig. 1). As transecções que atravessavam os cordões de dunas no sentido continente-mar (números 1 a 3 ) distavam entre si cerca de $3 \mathrm{~km}$ e tinham aproximadamente $3 \mathrm{~km}$ de extensão.

A transecção delimitada ao longo da costa (número 4) foi estabelecida porque nem todas as transecções atingiam as dunas da praia e, quando as atingiam, não permitiam uma boa amostragem desse habitat. Essa transecção percorria as dunas da praia apenas por uma extensão aproximada de $5 \mathrm{Km}$.

Cada transecção foi percorrida uma vez por mês, geralmente das 8 às 15 horas. Durante o percurso, a aproximadamente cada dez metros, caminhava-se cinco metros para cada lado da picada.

As transecções de sentido continente-mar permitiram a realização de coletas nos diferentes habitats existentes: tabuleiro, matas costeiras entre-dunas, dunas fixas com vegetação de restinga e dunas costeiras. No tabuleiro, situado na encosta oeste 
das dunas, a procura dos espécimes foi efetuada sob moitas arbóreas e sob pedras. Nas matas costeiras entre-dunas, as coletas foram feitas no folhiço, junto a ou sob os troncos em decomposição e nos galhos das árvores. A procura dos espécimes sob o folhiço foi feita com auxílio de um ancinho e a captura foi manual. As coletas junto aos troncos em decomposição também foram manuais, utilizando-se ancinho e pá para revirá-los. As espécies arborícolas foram capturadas com garrucha calibre 22 , munida com chumbo mostarda.

$\mathrm{Na}$ vegetação de restinga das dunas fixas, coletas manuais foram efetuadas nas touceiras de bromeliáceas, no folhiço sob moitas arbóreo-arbustivas e na vegetação herbácea. Nas dunas costeiras, as coletas foram feitas nos troncos em decomposição sobre a areia, na vegetação herbácea e nas moitas arbóreo-arbustivas.

Na restinga de Ponta de Campina, duas transecções no sentido continentemar foram percorridas uma vez por mês, geralmente das 8 às 15 horas. Como a área era pequena ( $1.000 \mathrm{~m}$ de largura por $900 \mathrm{~m}$ de comprimento) e fisionomicamente pouco heterogênea, as transecções, escolhidas aleatoriamente à direita e à esquerda da via de acesso à praia, permitiram uma boa amostragem dos vários habitats. Ao longo das transecções, foram observados e/ou coletados espécimes sob moitas arbóreo-arbustivas, dentro de bromeliáceas e na vegetação herbácea. Na área de mata, as coletas foram feitas no folhiço, nos troncos em decomposição e nos galhos das árvores. Apenas para a captura de espécies arborícolas e de grandes espécimes no chão da mata utilizou-se garrucha; as demais espécies foram coletadas manualmente, com auxílio de ancinho.

Para cada observação/coleta, foram anotados o habitat, o microhabitat, a data, a hora e o número de observações. As anotações sobre os locais de coleta permitiram conhecer o habitat utilizado pelas espécies (mata ou áreas abertas) e o seu modo de distribuição nesses habitats. Tais informações auxiliaram no conhecimento sobre a organização dessas comunidades de lagartos.

Os espécimes obtidos foram acondicionados em sacos plásticos e etiquetados com número de campo. Em laboratório, foram fixados com formol a $10 \%$ e posteriormente mantidos em álcool a 70\%. Todos os espécimes estão depositados na coleção herpetológica do Departamento de Sistemática e Ecologia, Universidade Federal da Paraíba.

Para a identificação das espécies, foi utilizada a literatura taxonômica pertinente, o que foi suplementado pela comparação com espécimes previamente identificados por especialistas e depositados nas coleções herpetológicas do Museu de Zoologia da Universidade de São Paulo e da Universidade Federal da Paraíba.

\section{RESULTADOS E DISCUSSÃO}

Foram observados 376 espécimes (13 espécies de lagartos e uma de anfisbena) nos diversos habitats e microhabitats das dunas de Natal e 184 espécimes (11 espécies de lagartos) em Ponta de Campina; destes, foram coletados 238 e 56 espécimes, respectivamente, de todas as espécies obervadas.

As tabelas I e II mostram a lista das espécies anotadas para cada localidade, o número de exemplares observados e/ou coletados e os dados sobre habitat e microhabitat. 
Tabela I. Lista de espécies e número de exemplares observados e/ou coletados nos diversos habitats e microhabitats das dunas de Natal, Rio Grande do Norte, durante 14 meses, nos anos de 1984 a 1986.

\begin{tabular}{|c|c|c|c|c|c|c|c|c|c|c|c|c|c|c|c|c|c|}
\hline \multirow{3}{*}{ Familia } & \multirow{3}{*}{ Espécie } & \multicolumn{16}{|c|}{ Habitats e microhabitats } \\
\hline & & \multicolumn{4}{|c|}{ Mata interdunar } & \multicolumn{4}{|c|}{ Restinga } & \multicolumn{7}{|c|}{ Dunas da praia } & \multirow{2}{*}{$\frac{\text { Tabuleiro }}{M}$} \\
\hline & & A & B & C & D & $E$ & $\mathrm{~F}$ & G & $\mathrm{H}$ & A & C & $\mathrm{F}$ & G & 1 & $\mathrm{~J}$ & $L$ & \\
\hline \multirow{5}{*}{ Gekkonidae } & Briba brasiliana & - & - & - & - & 8 & - & - & - & - & - & - & - & - & - & - & - \\
\hline & Coleodactylus cf. meridionalis & - & 40 & - & - & 1 & - & - & - & - & - & - & - & - & - & - & - \\
\hline & Gymnodactylus darwinii & 12 & 1 & - & 4 & - & - & - & - & - & - & - & - & - & - & - & - \\
\hline & Gymnodactylus geckoides & - & - & - & - & 20 & - & - & - & 49 & - & - & 3 & - & - & 2 & 7 \\
\hline & Hemidactylus mabouia & 8 & 1 & 4 & - & 5 & - & - & - & 40 & - & - & - & - & - & 3 & - \\
\hline Polychrotidae & Enyalius bibronii & - & - & 1 & - & - & - & - & - & - & - & - & - & - & - & - & - \\
\hline Tropiduridae & Tropidurus hispidus & 1 & 20 & 3 & - & 6 & 20 & 1 & - & 10 & - & 12 & 6 & 3 & - & - & 1 \\
\hline \multirow[t]{3}{*}{ Teiidae } & Ameiva ameiva & - & 13 & - & - & - & 5 & 3 & - & - & - & 2 & - & - & - & - & - \\
\hline & Anotosaura sp. & - & 8 & - & - & - & - & - & - & - & - & - & - & - & - & - & - \\
\hline & Cnemidophorus ocellifer & - & - & - & - & - & 8 & 2 & - & - & - & 5 & 18 & - & - & 1 & - \\
\hline Gymnophthalmidae & Micrablephanus maximiliani & - & 3 & - & - & 3 & 2 & - & - & - & - & 1 & - & - & - & - & - \\
\hline Scincidae & Mabuya macrorhyncha & - & - & - & - & 6 & - & - & - & - & - & - & - & - & - & - & - \\
\hline Anguidae & Diplogossus lessonae & - & 2 & - & - & - & - & - & - & - & - & - & - & - & - & - & - \\
\hline Amphisbaenidae & Amphisbaena heathi & - & - & - & - & - & - & - & 1 & - & - & - & - & - & - & - & 1 \\
\hline
\end{tabular}

(A) Troncos em decomposição, (B) folhiço, (C) galhos ou troncos de árvores, (D) buracos em troncos de árvores ou entre raizes tabulares, $(E)$ bromélias, $(F)$ sob moita arbustiva, $(G)$ vegetação herbácea, $(\mathrm{H})$ subterrâneo, $(\mathrm{I})$ areia nua, $(\mathrm{J})$ sob moita arbórea, $(\mathrm{L})$ cactáceas em decomposição, (M) sob pedras no tabuleiro.

Tabela II. Lista de espécies e número de exemplares observados e/ou coletados nos habitats e microhabitats da restinga de Ponta de Campina e da mata (BR-230, Km 11), Cabedelo, Paraíba, durante 14 meses, nos anos de 1984 a 1986.

\begin{tabular}{|c|c|c|c|c|c|c|c|c|c|}
\hline \multirow{3}{*}{ Familia } & \multirow{3}{*}{ Espécie } & \multicolumn{8}{|c|}{ Habitats e microhabitats } \\
\hline & & \multicolumn{4}{|c|}{ Mata interdunar } & \multicolumn{4}{|c|}{ Restinga } \\
\hline & & A & B & C & D & $\mathrm{E}$ & $\mathrm{F}$ & G & $\mathrm{J}$ \\
\hline \multirow[t]{3}{*}{ Gekkonidae } & Coleodactylus meridionalis & - & 7 & - & - & - & - & - & 3 \\
\hline & Gymnodactylus darwinii & 10 & 1 & - & 5 & - & - & - & - \\
\hline & Hemidactylus mabouia & - & - & - & - & 2 & - & - & - \\
\hline \multirow[t]{2}{*}{ Polychrotidae } & Anolis fuscoauratus & - & 1 & 4 & - & - & - & - & - \\
\hline & Anolis ortonii & - & - & 2 & - & - & - & - & - \\
\hline Tropiduridae & Tropidurus hispidus & - & 1 & 2 & 1 & 3 & 20 & 7 & - \\
\hline \multirow[t]{3}{*}{ Teiidae } & Ameiva ameiva & - & 3 & - & - & - & 10 & - & 4 \\
\hline & Anotosaura sp. & - & 4 & - & - & - & - & - & - \\
\hline & Cnemidophorus ocellifer & - & - & - & - & - & 46 & 25 & 15 \\
\hline Gymnophthalmidae & Micrablepharus maximiliani & - & - & - & - & - & 1 & - & - \\
\hline Scincidae & Mabuya macrorhyncha & - & - & - & - & 7 & - & - & - \\
\hline
\end{tabular}

(A) Troncos em decomposição, (B) Folhiço, (C) galhos ou troncos de árvores, (D) buracos em troncos de árvores ou entre raizes tabulares, $(E)$ bromélias, $(F)$ sob moita arbustiva, $(G)$ vegetação herbácea, $(\mathrm{J})$ sob moita arbórea.

\section{Espécies de floresta}

Das nove espécies de lagartos registradas para a mata interdunar de Natal (Tab. I), apenas três (Coleodactylus cf. meridionalis, Gymnodactylus darwinii e Enyalius bibronii) são realmente animais de floresta. De modo similar, das sete obtidas neste habitat, em Cabedelo, apenas quatro (Coleodactylus meridionalis, Gymnodactylus darwinii, Anolis fuscoauratus e Anolis ortonii) são tipicamente habitantes de floresta. 
As demais espécies encontradas nestas matas são na realidade formas heliófilas, ou naturais de áreas abertas, que ocasionalmente penetram na floresta, onde não permanecem por não encontrarem sítios de termorregulação adequados.

Coleodactylus meridionalis (Boulenger,1888), conhecido da Mata Atlântica e de alguns brejos florestados (VANZOLINI 1957, 1968a, 1970, 1972), foi posteriormente encontrado no sertão de Pernambuco e na Chapada do Araripe, regiões muito menos úmidas que as demais localidades de onde era conhecido (VANZOLINI 1980, 1981; VANZOLINI et al. 1980).

Dos 40 espécimes de $C$. cf. meridionalis obtidos na mata das dunas de Natal, apenas cinco $(12,5 \%)$ estavam sob folhiço da mata interdunar alta, com árvores de 15 ou mais metros de altura. Os demais espécimes foram obtidos sob o folhiço da mata baixa, com árvores de três a sete metros de altura. Na mata baixa, $C$. cf. meridionalis foi encontrado tanto sob folhiço profundo próximo aos troncos de árvores, como em solo com folhiço mais esparso em áreas mais abertas (Tab. I). Foram encontrados três ovos desta espécie (um de cada vez), sob folhiço da mata interdunar baixa. Dos dois coletados em 20/05/85, um eclodiu em 20/06 e outro em 20/07.

O único exemplar de C. cf. meridionalis obtido na vegetação de restinga das dunas fixas foi encontrado sobre areia, entre touceiras de bromeliáceas terrestres (Hohembergia ramageana Mez) (Tab. I).

A presença de C. cf. meridionalis principalmente na mata baixa, bem como na vegetação de restinga, mostra que a espécie pode tolerar ambientes pouco sombreados. Como nas dunas a tendência é a regressão da mata, a ocupação de outros habitats pode ser característica pré-adaptativa e talvez venha a assegurar a permanência do lagarto nesse ecossistema.

Dos 10 espécimes de C. meridionalis obtidos em Cabedelo, sete foram encontrados na mata e três na restinga (Tab. II). Na mata, C. meridionalis estava sob folhiço profundo; na restinga foi encontrado no folhiço de cajueiro (Anacardium occidentale). A presença desta espécie na restinga deve estar relacionada com a progressiva devastação da mata de Cabedelo, que anteriormente estendia-se tanto para o norte, como para o sul, até o Estuário do Rio Paraíba. Com a destruição de parte dessa mata, estes lagartos passaram a ocupar também a restinga contígua a esta, em microhabitat semelhante, embora em condições mais secas.

A população de Coleodactylus das dunas de Natal mostra diferenças acentuadas, principalmente quanto ao padrão de colorido, quando comparada à espécie de ampla ocorrência na costa do Nordeste (Coleodactylus meridionalis). Os espécimes obtidos em Cabedelo concordam, em todos os caracteres diagnósticos, com C. meridionalis. Os problemas envolvidos na diferenciação da população das dunas de Natal serão discutidos futuramente.

Gymnodactylus darwinii (Gray,1845), conhecido inicialmente apenas da Mata Atlântica do sul da Bahia ao litoral norte de São Paulo (VANZOLINI 1953, 1968a, 1968b, 1972), foi posteriormente encontrado na Mata Atlântica do Nordeste (VANZOLINI 1974). São atualmente conhecidas várias populações de áreas abertas em Cabo Frio, Rio de Janeiro e restinga de Barra de Maricá (ARAúJo 1985). Na 
mata, $G$. darwinii é encontrado sob detritos vegetais, em troncos de árvores e sob troncos em decomposição (VANZOLINI 1953, 1972, 1974).

Dezessete exemplares de Gymnodactylus darwinii foram obtidos na mata das dunas de Natal, geralmente dentro, sob ou sobre troncos em decomposição (Tab. I). Os troncos onde $G$. darwinii foi encontrado estavam sempre colonizados por térmites e com muitos detritos vegetais, microhabitat abundante na mata alta onde a espécie foi geralmente coletada.

Habitando troncos semelhantes aos utilizados por $G$. darwinii, foi encontrado Hemidactylus mabouia. Num mesmo tronco, foram encontrados dois adultos e um filhote de $G$. darwinii, juntamente com um ovo de $H$. mabouia.

As baixas densidades nas populações de $G$. darwinii no litoral paulista (VANZOLINI 1953) e em Barra de Maricá (ARAúJO 1985) foram atribuídas à competição com H. mabouia. Segundo SCHOENER (1974), uma das combinações mais comuns nas comunidades aminais é a de espécies que se sobrepõem em habitat para comer diferentes alimentos. Um exemplo clássico foi observado nos Anolis de Bimini, onde várias espécies utilizam o mesmo habitat, alimentando-se de presas diferentes (SCHOENER 1968). Uma análise do conteúdo estomacal de G. darwinii e H. mabouia esclareceria se eles se sobrepõem apenas na utilização do espaço.

Um dado da maior importância que este estudo permitiu obter diz respeito ao contato, nas dunas de Natal, entre $G$. darwinii e $G$. geckoides. Nas dunas da praia, a aproximadamente 10 metros dos vales de mata onde $G$. darwinii é encontrado, a espécie presente é $G$. geckoides, que habita troncos similares, porém nas áreas abertas. Têm-se, assim, as duas espécies em simpatria, embora não em sintopia, nas dunas de Natal. Essa distribuição pode estar relacionada a adaptações desenvolvidas antes da simpatria, como sugerido por HUEY (1979), uma vez que G. geckoides e G. darwinii ocupam nichos similares quando em alopatria (VANZOLINI et al. 1980; VANZOLINI 1982).

Em Cabedelo, foram obtidos dezesseis espécimes de $G$. darwinii, todos na mata (Tab. II). De modo similar ao que se verifica em Natal, eles preferem troncos em decomposição no folhiço, também com cupinzeiro. Em menor escala, foi encontrado sobre troncos de árvores e entre raizes tabulares (Tab. II). Dois ovos encontrados dentro de tronco em decomposição em 03/07/85 eclodiram em 09/07/85.

Os Gymnodactylus encontrados na mata das dunas de Natal, aqui tratados como G. darwinii, mostram problema similar ao dos Coleodactylus. A população de Natal apresenta diferenciação morfológica acentuada e trata-se, possivelmente, de uma nova forma. Este problema será analisado futuramente.

O gênero Enyalius Wagler distribui-se amplamente na Mata Atlântica (ETHERIDGE 1969; VANZOLINI 1972, 1974; JACKSON 1978), ocorrendo desde o Estado do Rio Grande do Sul até Pernambuco. Neste Estado é encontrado, também, em ilhas de mata mais para o interior do continente (JACKSON 1978). O gênero ainda inclui uma espécie amazônica (JACKSON 1978).

Apenas um espécime de Enyalius foi obtido na mata das dunas de Natal, em dia nublado, após chuva, sobre galho de árvore a aproximadamente um metro de 
altura. Embora seja necessária uma nova revisão para melhor caracterizar as espécies do gênero, o espécime coletado em Natal pode, por enquanto, ser atribuído a Enyalius bibronii Boulenger, 1885.

Apesar de três transecções atravessarem vales de mata entre-dunas, nenhum outro espécime foi encontrado. JACKSON (1878) atribui o pequeno tamanho de sua amostra ao fato das espécies de Enyalius (exceto bilineatus) ocorrerem em populações de baixa densidade. Uma vez que Enyalius bibronii ocorre em diferentes microhabitats, como galhos, troncos de árvores e arbustos em áreas abertas (JACKSON 1978), talvez a aparente raridade desta espécie na área seja real.

As espécies de Anotosaura Amaral, 1933 têm sido encontradas principalmente em áreas abertas, em localidades de caatinga (VANZOLINI \& RAMOS 1977; RODRIGUES 1986), cerrado, agreste e em áreas de interdigitações de caatinga e cerrado (DiXON 1974; VANZOLINI 1974, 1976b; VANZOLINI \& RAMOS 1977). No entanto, poucas são as informações acerca de suas preferências ecológicas. $\mathrm{Na}$ caatinga em Cabaceiras, Paraíba, Anotosaura vanzolinia, espécie descrita do agreste, é encontrada apenas em ambientes mais mésicos que a caatinga aberta (RODRIGUES 1986).

Anotosaura sp., encontrada inicialmente na Mata Atlântica em João Pessoa, Paraíba e em Brejo dos Cavalos, Pernambuco (Miguel T. Rodrigues, comunicação pessoal), foi obtida em Natal e em Cabedelo. Em ambas as áreas, foi encontrada apenas na mata, principalmente sob folhiço profundo e úmido próximo aos troncos das árvores ou entre as raízes tabulares. Esta espécie ainda não foi descrita.

Duas espécies de Anolis foram obtidas em Cabedelo: Anolis fuscoauratus Duméril \& Bibron, 1837 e Anolis ortonii Cope, 1869 (Tab. II). Anolis fuscoauratus é espécie arborícola que ocorre nas Florestas Amazônica e Atlântica, do nordeste da Colômbia ao Estado do Rio de Janeiro (VANZOLINI 1974). Apesar de ser principalmente arborícola e frequente na floresta primária (BEEBE 1944b), Anolis fuscoauratus é abundante em matas secundárias, ao longo de clareiras e no folhiço (VANZOLINI 1972, 1974; DiXON \& SOINI 1975; CUNHA et al. 1985).

Em Cabedelo, a espécie foi observada em troncos de árvores com aproximadamente oito a dez centímetros de diâmetro, entre dois e quatro metros de altura, na mata secundária. Um único espécime foi visto no folhiço, de onde escapou para um tronco de árvore, subindo a mais de quatro metros de altura (Tab. II).

Anolis ortonii também é arborícola, tendo sido observado em alturas de até oito metros (DIXON \& SoINI 1975), em vegetação secundária, bordas, clareiras e até em áreas cultivadas (VANZOLINI 1972; DiXON \& SoINI 1975; Duellman 1978; CUNHA et al. 1985). Na mata de Cabedelo, esta espécie foi encontrada em tronco de árvore com aproximadamente $10 \mathrm{~cm}$ de diâmetro, a cerca de três metros de altura. Oito ovos foram encontrados no tronco de uma árvore com térmites, em 31-V-85. Esses ovos estavam depositados em buracos no tronco e no cupinzeiro, a cerca de $50 \mathrm{~cm}$ de altura. Mantidos em recipientes com pedaços de tronco e folhiço, cinco ovos eclodiram: um no dia da coleta, dois no dia 09/06, o quarto no dia 22/06 e o quinto em 29/06.

Ninhos comunais, tanto intra, como inter-específicos, foram comumente 
encontrados na região de Iquitos, no Peru; muitos pertencentes a Anolis ortonii (DiXON \& SoINI 1975). A maioria das ninhadas foi vista em formigueiros, que apresentavam condições para a incubação dos ovos, protegendo-os, inclusive, da predação.

Nenhuma espécie de Anolis foi encontrada em Natal. Uma vez que Anolis fuscoauratus e $A$. ortonii, apesar de arborícolas, são bastante tolerantes às mais diversas situações da mata, fica difícil justificar suas ausências na mata das dunas de Natal, onde, aparentemente, tem-se maior disponibilidade de habitat que na mata de Cabedelo.

A pequena amostragem e até ausência de espécies arborícolas na mata das dunas de Natal, comparada com a boa amostragem de outras espécies obtidas no folhiço e nos troncos em decomposição, talvez possa ser justificada pela metodologia de coleta. $\mathrm{O}$ folhiço e os troncos eram mexidos e revirados durante as coletas, enquanto que as espécies arborícolas eram procuradas nas árvores ao longo das transecções. A mesma metodologia foi utilizada na mata de Cabedelo, mas essa área era bem menor, mais aberta, facilitando a observação dos lagartos nos galhos. É provável que, em coletas futuras nas matas das dunas, espécies arborícolas sejam encontradas com mais frequência.

\section{Espécies de áreas abertas}

Em Natal, oito espécies de lagartos e uma de anfisbena de áreas abertas foram obtidas nas dunas ao longo da costa e na vegetação de restinga, sobre cordões de dunas fixas mais interiores (Tab. I). Em Ponta de Campina, os lagartos de áreas abertas foram coletados apenas na restinga (sete espécies), uma vez que a região de praia encontrava-se loteada (Tab. II).

Das espécies obtidas nos dois locais de estudo, Tropidurus hispidus (Spix, 1825) foi a única a frequentar todos os habitats e praticamente todos os microhabitats disponíveis (Tabs I, II), confirmando sua posição como generalista de habitat (RODRIGUES 1987). Outras espécies que também ocupam vários habitats nas dunas de Natal são Hemidactylus mabouia, Ameiva ameiva e Micrablepharus maximilia$n i$.

Um aspecto óbvio da diferença entre as espécies das áreas abertas das dunas de Natal e da restinga de Ponta de Campina, é a presença marcante de geconídeos nas dunas. Em Natal, foram obtidas três espécies de geconídeos de formações abertas (Briba brasiliana, Gymnodactylus geckoides e Hemidactylus mabouia); os dois últimos bastante abundantes (Tab. I). Em Ponta de Campina, foi encontrada apenas H. mabouia, aparentemente, em baixa densidade (Tab. II).

Briba brasiliana Amaral, 1935 encontra-se distribuída em formações abertas, do sul do Piauí ao Vale do São Francisco/MG. É encontrada tanto na caatinga (VANZOLINI 1974, 1981; VANZOLINI et al. 1980; RoDRIGUES 1986), como no cerrado (VANZOLINI 1968a, 1974, 1976a). Até o presente, sua ocorrência em localidades costeiras era desconhecida. Nas dunas de Natal, B. brasiliana foi encontrada apenas nas dunas fixas com vegetação de restinga, dentro de touceiras da bromeliácea terrestre Hohembergia ramageana Mez. Foram coletados vários exemplares em uma mesma bromélia, ou em associação com Mabuya macrorhyn- 
cha e G. geckoides. Esta espécie também foi vista sob bromélias desfolhadas na areia, juntamente com $T$. hispidus.

Outro geconídeo abundante nas áreas abertas das dunas de Natal foi Gymnodactylus geckoides Spix, 1825 (Tab. I). Esta espécie é habitante das caatingas do Nordeste, da Bahia ao Rio Grande do Norte (VANZOLINI 1953, 1968a, 1968b, 1970, 1974, 1982; VANZOLINI et al. 1980), onde é geralmente encontrada sob ou dentro de troncos em decomposição e sob pedras.

Nas dunas de Natal, $G$. geckoides habita a vegetação de restinga, o sopé das dunas e as dunas da praia, predominando na última (Tab. I). Na vegetação de restinga, foi observado somente em touceiras da bromeliácea Hohembergia ramageana, sendo o lagarto mais abundante nesse microhabitat, onde foi avistado, uma das vezes, às 12:30 hs, exposto ao sol. Embora pouco seja conhecido sobre o comportamento de geconídeos noturnos durante o dia (BRATTSTROM 1952; PIANKA \& HUEY 1978), algumas observações de campo sugerem que esses lagartos talvez se exponham ao sol, durante o dia, para se aquecer (BRATTSTROM 1952).

Em uma mesma touceira, G. geckoides foi encontrado junto com Mabriya macrorhyncha e Briba brasiliana. Folhas de touceiras desmanchadas foram utilizadas como abrigo, ao mesmo tempo, pôr G. geckoides, Micrablepharus maximiliani e Tropidurus hispidus. Devido à capacidade de armazenar água, as bromeliáceas, além de abrigarem vários grupos de invertebrados, constituem um excelente microhabitat para outros vertebrados, incluindo os lagartos (SMITH 1941; РEIXOTO 1977). Nessa área de vegetação de restinga, com ambiente bastante seco e moitas arbustivas esparsas, as bromeliáceas constituem uma das poucas fontes de abrigo e alimento. A grande quantidade de bromélias permite a coexistência de diferentes espécies de lagartos, normalmente habitantes das caatingas, onde a disponibilidade de recursos é ainda menor.

No tabuleiro, G. geckoides foi encontrado sob pedras. Nas dunas da praia, na ausência de bromeliáceas e de pedras, a espécie ocupa os troncos em decomposição sobre a areia, onde novamente é o lagarto mais abundante. Foram obtidos até três exemplares em um mesmo tronco; na maioria das vezes, a espécie foi vista juntamente com H. mabouia. A grande quantidade de troncos em decomposição, com muitos cupins e formigas, constitui-se num dos poucos microhabitats disponíveis e abundantes, em amplas áreas de areia nua, capaz de fornecer abrigo, proteção contra predação e, provavelmente, alimento. Novamente, a abundância desse microhabitat pode justificar a coexistência de G. geckoides, H. mabouia e T. hispidus.

Hemidactylus mabouia (Moreau de Jonnès, 1818), um geconídeo introduzido da África e muito comum em ambientes periantrópicos (GoELDI 1902; BEEBE 1944a; CUNHA 1961; HoOGMOED 1973; VANZOLINI 1968a, 1978; VANZOLINI et al. 1980), ocorre, devido à sua grande habilidade de colonização, na área nuclear de todos os dominíos morfoclimáticos do Brasil e em áreas transicionais (VANZOLINI 1978). Essa habilidade foi confirmada nas dunas de Natal, onde a espécie foi encontrada em todos os habitats, predominando nas áreas abertas, especialmente nos troncos em decomposição sobre as dunas da praia (Tab. I). Tanto nesses troncos, quanto nas bromeliáceas da vegetação de restinga, é encontrado principalmente com G. geckoides. 
A presença e abundância de.geconídeos em Natal, contraposta à baixa densidade ou ausência de algumas espécies em Cabedelo, poderia estar relacionada com a maior heterogeneidade espacial das dunas (PIANKA 1966, 1969; HEYER 1967; PIANKA \& HUEY 1978). No entanto, em habitats similares, essa correlação parece ser devida muito mais à dependência de algumas espécies a determinados microhabitats do que à relação fauna-flora (HEYER 1967). Nesse caso, não haveria como justificar a ausência de alguns geconídeos na restinga de Ponta de Campina, onde há grande disponibilidade de bromélias.

Em busca de uma explicação para esta diferença tão acentuada, procurou-se avaliar em que medida a proximidade geográfica das caatingas contribuiu para este padrão. Comparou-se a fauna de lagartos obtida nas dunas de Natal e na restinga de Ponta de Campina com aquela descrita para as caatingas (Tab. III). Das 17 espécies típicas das caatingas, 9 (53\%) foram encontradas nas dunas de Natal, enquanto apenas 6 (35,3\%) estão presentes na restinga de Ponta de Campina (Tab. III). Das espécies ausentes em Ponta de Campina (Briba brasiliana, Gymnodactylus geckoides e Diploglossus lessonae), principalmente as duas primeiras são formas típicas de caatinga (VANZOLINI 1974, 1976a, 1981; RoDRIGUES 1986). Fica claro que existe semelhança entre a fauna das caatingas e a das dunas de Natal e restingas de Ponta de Campina, sendo bem maior no primeiro caso. Como a caatinga está mais próxima do litoral no Rio Grande do Norte do que na Paraíba, a maior influência da fauna das caatinga nas dunas deve ser resultante da proximidade geográfica entre esses Ecossitemas.

Tabela III. Ocorrência de lagartos nas caatingas (dados de VANZOLINI 1974; RODRIGUES 1986) e nas áreas abertas das dunas de Natal, Rio Grande do Norte e da restinga de Ponta de Campina, Cabedelo, Paraiba.

\begin{tabular}{lccc}
\hline \multicolumn{1}{c}{ Espécies } & Caatinga & Dunas & Restinga \\
\hline Briba brasiliana & + & + & - \\
Gymnodactylus geckoides & + & + & - \\
Hemidactylus mabouia & - & + & + \\
Phyllopezus periosus & + & - & - \\
Phyllopezus pollicaris & + & - & - \\
Vanzoia klugei & + & - & - \\
lguana iguana & + & - & - \\
Polychrus acutirostris & + & + & - \\
Tropidurus hispidus & + & - & + \\
Tropidurus semitaeniatus & + & + & + \\
Ameiva ameiva & + & - & - \\
Colobosaura mentalis & + & + & + \\
Cnemidophorus ocellifer & + & - & + \\
Vanzosaura rubricauda & + & + & + \\
Micrablepharus maximiliani & + & + & + \\
Tupinambis teguixin & + & + & - \\
Mabuya macrorhyncha & + & + & - \\
Diploglossus lessonae & + & + & + \\
Amphisbaena heathi & - & & \\
\hline
\end{tabular}


Ameiva ameiva (Linnaeus,1758) e Cnemidophorus ocellifer (Spix, 1825), dois teiídeos de ampla distribuição geográfica, principalmente nas formações abertas, foram obtidos em Natal e Ponta de Campina (Tabs I, II). Em Natal, além de habitar as áreas francamente abertas, $A$. ameiva foi encontrado principalmente nas bordas e clareiras da mata interdunar. Na vegetação de restinga, como em Ponta de Campina, $C$. ocellifer e A. ameiva utilizam habitats similares, diferindo quanto à utilização do microhabitat. C. ocellifer predomina nas bordas das moitas arbustivas, A. ameiva é mais encontrado no centro das moitas. Esse padrão difere do observado na restinga de Barra de Márica, onde A. ameiva prefere as bordas das moitas (ARAÚJO 1985). Além de ocuparem habitats semelhantes nas áreas abertas, $C$. ocellifer e A. ameiva utilizam estratégia de forrageamento similar, do tipo "procurador" (HuEY \& PIANKA 1981; MAGNUSSOM et al. 1985); no entanto, A. ameiva parece utilizar uma maior área para o forrageamento (MAGNUSSOM et al. 1985). Essas diferenças na utilização do microhabitat e na intensidade de forrageamento devem auxiliar na partilha de recursos, mas precisam ser estudadas quantitativamente, com métodos diferentes dos utilizados neste trabalho.

Micrablepharus maximiliani (Reinhardt \& Lütken, 1862), um microteiídeo que habita toda a diagonal de formações abertas (VANZOLINI \& RAMOS 1977), mas parece preferir climas mais amenos que o da caatinga aberta (VANZOLINI et al. 1980; VANZOLINI 1981), foi encontrado em Natal, principalmente nas áreas abertas, embora penetre na mata, onde vive no folhiço (Tab. I). Na restinga de Ponta de Campina, somente um espécime foi encontrado, no folhiço sob moita arbustiva.

Mabuya macrorhyncha Hoge, 1946, descrita da Ilha de Queimada Grande, São Paulo, tem sido encontrada principalmente nas restingas da costa leste do Brasil (HoGE 1946; REBOUÇAS-SPIEKER 1974; VANZOLINI \& GOMES 1979; ARAÚJO 1985). Essa espécie era desconhecida das caatingas, mas uma forma morfologicamente muito próxima foi encontrada recentemente em Cabaceiras (RODRIGUES 1986; STEVAuX 1993).

Nas restingas, $M$. macrorhyncha habita touceiras de bromeliáceas terrestres do gênero Hohembergia (VANZOLINI \& GoMES 1979) e Neoregelia cruenta (ARAÚJo 1985). Na caatinga, apesar de algumas vezes ser vista em troncos ou sob casca de facheiros, M. macrorhyncha vive, principalmente, em touceiras de macambira (Bromelia lacinosa) sobre os lajedos (RODRIGUES 1986).

Tanto nas dunas de Natal, como na restinga de Ponta de Campina, Mabuya macrorhyncha foi encontrada apenas na vegetação de restinga, dentro de touceiras da bromeliácea terrestre Hohembergia ramageana (Tabs I, II).

Em Ponta de Campina, Mabuya macrorhyncha foi vista sobre folha de bromélia, sempre durante ou logo após a chuva. Fato semelhante, observado na restinga de Márica, levou à hipótese de que sua atividade possa estar mais relacionada com a umidade do ambiente do que com a tempertura (ARAúJO 1985).

Apenas um Anfisbenídeo, Amphisbaena heathi Schmidt, 1936 foi coletado nas dunas de Natal; esta espécie foi descrita de Baixa Verde, atualmente João Câmara, Rio Grande do Norte (ScHMidT 1936). Posteriormente, foi efetuada uma redescrição da espécie (GANS 1965), no entanto não se tem informações sobre as suas preferências ecológicas. Até então, ela era conhecida apenas da localidade tipo. 
Amphisbaena heathi foi encontrada em Natal em áreas abertas, na vegetação de restinga e no tabuleiro. Na restinga foi encontrada sob a areia úmida entre as raizes de touceiras da bromeliácea $H$. ramageana. $\mathrm{O}$ exemplar obtido no sopé das dunas estava sob pedras, em microhabitat similar ao utilizado por G. geckoides.

\section{CONCLUSÕES}

A fauna de lagartos das dunas de Natal e da restinga de Ponta de Campina é constituída por espécies ecologicamente ligadas às formações abertas e por espécies de floresta. A maioria das espécies obtidas nas áreas abertas das dunas e da restinga faz parte da fauna da grande diagonal de formações abertas, que se estende do nordeste da Argentina ao Maranhão. Além disso, existe semelhança entre a fauna de lagartos das dunas e da restinga e aquela descrita para as caatingas, bem maior no primeiro caso, mostrando que a influência da fauna das caatingas varia com a distância entre esses Ecossistemas.

As espécies de lagartos típicas de florestas, encontradas na mata das dunas de Natal e da restinga de Ponta de Campina são todas pertencentes à fauna da Mata Atlântica, embora a maioria das espécies das dunas encontrem-se, morfologicamente, bastante diferenciadas.

Gymnodactylus darwinii e G. geckoides são espécies simpátricas, embora não sintópicas, nas dunas de Natal; o primeiro vive na mata interdunar e o segundo nas áreas abertas (restingas e dunas da praia).

AGRADECIMENTOS. Ao Dr. Miguel Trefaut Rodrigues (Departamento de Zoologia, Universidade de São Paulo - USP), pela orientação, apoio e incentivo durante este trabalho. Ao Dr. Paulo Emílio Vanzolini (Museu de Zoologia, USP), pelas facilidades no acesso à Coleção Herpetológica daquela Instituição e fornecimento de separatas de suas publicações. Às doutoras Norma Gomes, Regina Rebouças-Spieker e Ana Maria Ramos-Costa (Museu de Zoologia, USP). Ao biólogo Roberto Lima Santos (Universidade Federal do Rio Grande do Norte), pelo auxílio nas coletas nas dunas. Aos doutores Gustavo Martinelli (Jardim Botânico do Rio de Janeiro) e Marcelo Ataíde (Empresa Pernambucana de Pesquisa Agropecuária) que identificaram as espécies vegetais. A Sra. Dione Seripierre (Biblioteca do Museu de Zoologia, USP) pelo auxílio na obtenção de bibliografia. À CAPES pela concessão da bolsa de Mestrado, que possibilitou a realização deste trabalho.

\section{REFERÊNCIAS BIBLIOGRÁFICAS}

Andrade-Lima, D. DE. 1961. Tipos de Floresta de Pernambuco. Anais Ass. Geógr. bras. 12 (1): $69-85$.

ARAÚJo, A.F.B. DE. 1985. Partilha de recursos em uma guilda de lagartos de restinga (Sauria). Dissertação de Mestrado, não publicada, Instituto de Biociências, Universidade de Campinas, Campinas, 113p.

ARAÚJo, D.S.D. \& R.P.B. HENRIQUES. 1984. Análise florística das restingas do Estado do Rio de Janeiro, p.159-193. In: L.D. LACERDA; D.S.D. ARAÚJO; R. CERQUeIrA \& B. TURCQ (Eds). Restingas: origem, estrutura e processos. 
Niterói, Universidade Federal Fluminense, 400p.

BEEBE, W. 1944a. Field notes on the lizards of Kartabo, British Guiana and Caripito,

Venezuela. Part 1. Gekkonidae. Zoológica 29: 145-160.

. 1944b. Field notes on the lizards of Kartabo, British Guiana and Caripito,

Venezuela. Part 2. Iguanidae. Zoológica 24: 195-215.

BRATTSTROM, B.H. 1952. Diurnal activities of a nocturnal animal. Herpetologica 8: $61-62$.

CASTRO, A.L. DE. 1952. Sobre a ocorrência do Gênero Tylos Latreille no litoral brasileiro (Isopoda, Tylidae). Bolm. Mus. Nac. Rio de Janeiro Zool. 107: 1-7.

CUNHA, O.R. DA. 1961. II. Lacertílios da Amazônia. Os lagartos da Amazônia

Brasileira, com especial referência aos representados na coleção do Museu

Goeldi. Bolm. Mus. paraense E. Goeldi Zool. 39: 1-189.

CunHA, O.R. DA; F.P. NASCIMENTO \& T.C.S. DE Ávila-PIRES. 1985. Os Répteis da área de Carajás, Pará, Brasil (Testudines e Squamata). Contrib. do Mus. paraense E. Goeldi ao Projeto Carajás 40: 1-92.

DiXON, J.R. 1974. Systematic review of the lizard genus Anotosaura (Teiidae). Herpetologica 30: 13-18.

DiXON, J.R. \& P. SorNI. 1975. The reptiles of the upper Amazon Basin, Iquitos

Region, Peru. I. Lizards and Amphisbaenians. Contr. Biol. Geol. 4: 1-58.

Duellman, W.E. 1978. The biology of an Equatorial Herpetofauna in Amazonian

Ecuador. Misc. Publs. Mus. nat. Hist. Univ. Kansas 65: 1-352.

Etheridge, R. 1969. A review of the iguanid genus Enyalius. Bull. Brit. Mus.

(Nat. Hist.) Zool. 18 (8): 233-260.

GANS, C. 1965. On Amphisbaena heathi Schmidt and A. carvalhoi, new species small forms from the northeast of Brasil (Amphisbaena: Reptilia). Proc. Calif. Acad. of Sci. 31 (23): 613-630.

GoELDI, E. 1902. Lagartos do Brasil. Bolm. Mus. paraense E. Goeldi 3: 499-560. Hallegouet, B. 1984. L'estuaire du Rio Paraiba. p.438-441. In: Geographie et ecologie de la Paraíba (Bresil). Travaux et Documents de Geographie Tropicale. Bordeaux, CEGET. 2 (50).

HAY, J.D.; R.P.B. HENRIQUES \& D.M. LIMA. 1981. Quantitative comparisons of dune and foredune vegetation in restinga ecosystems in the state of Rio de Janeiro, Brazil. Rev. Brasil. Biol. 41 (3): 655-662.

HEYER, W.R. 1967. A herpetofaunal study of an ecological transect through the Cordillera de Tilarán, Costa Rica. Copeia 2: 259-271.

Hoge, A.R. 1946. Um novo lagarto da Ilha da Queimada Grande. Mem. Inst.

Butantan 19: 241-248.

HoogmoEd, M. S. 1973. Notes on the herpetofauna of Surinam IV. The lizards and amphisbaenians of Surinam. Hague, W. Junk, 419p.

HUEY, R.B. 1979. Parapatry and niche complementarity of Peruvian desert geckos

(Phyllodactylus): the ambiguos role of competition. Oecologia 38 (3): 249-259.

HUEY, R.B. \& E.R. PIANKA. 1981. Ecological consequences of foraging mode.

Ecology 62 (4): 991-999.

JACKSON, J.F. 1978. Differentiation in the genera Enyalius and Strobilurus 
(Iguanidae): Implications for Pleistocene climatic changes in eastern Brazil. Arq. Zool., São Paulo, 30: 1-79.

KuhlmanN, E. 1977. Vegetação, p.85-106. In: Geografia do Brasil -Região Nordeste. Rio de Janeiro, Fund. Inst. Bras. Geogr. e Estatística (IBGE), vol. 2, $454 \mathrm{p}$.

LACERDA, L. D. DE; D.S.D. ARAÚJO \& N.C. MACIEL. 1982. Restingas brasileiras: uma bibliografia. Rio de Janeiro, Fund. José Bonifácio, 47p.

MACIEL, N.C. 1984. A fauna da restinga do Estado do Rio de Janeiro; passado, presente e futuro. Proposta de preservação, p.285-304. In: L.D. LACERDA; D.S.D. ARAÚJo; R. CERQUeIRA \& B. TURCQ (Eds). Restingas: origem, estrutura e processos. Niterói, Universidade Federal Fluminense, 400p.

Magnussom, W.E.; L.J. DE PAIVA; R.M. DA Rocha; C.R. FranKE; L.A. KasPeR \& A.P. LIMA. 1985. The correlates of foraging mode in a community of Brasilian lizards. Herpetologica 41 (3): 324-332.

NogueIRA, A.M.B. 1982. O Cenozóico continental da região de Natal-RN. Col. Textos Acad. UFRN 2 (284): 1-117.

NovaES, F.C. 1950. Sobre as aves de Sernambetiba, Distrito Federal, Brasil. Rev. Brasil. Biol. 10 (2): 199-208.

OTERO, L.S. 1984. Biologia de alguns tetigonídeos da restinga (Orthoptera; Tettigoniidae; Phaneropterinae), p.355-364. In: L.D. LACERDA; D.S.D. ARAÚJO; R. CERQUEIRA \& B. TURCQ (Eds). Restingas: origem, estrutura e processos. Niterói, Universidade Federal Fluminense.

PEIXOTO, O.L. 1977. Anfíbios anuros associados às bromeliáceas nos Estados do Rio de Janeiro e Espírito Santo. Dissertação de Mestrado, não publicada, Universidade Federal do Rio de Janeiro, 55p.

PIANKA, E.R. 1966. Latitudinal gradients in species diversity: a rewiev of concepts. Amer. Nat. 100: 33-46.

1969. Habitat specificity, speciation, and species diversity in Australian desert lizards. Ecology 50: 498-502.

PIANKA, E.R. \& R.B. HUEY. 1978. Comparative ecology, resource utilization and niche segregation among lizards gekkonid in the southern Kalahari. Copeia 4: 691-701.

PorTo, F.C.S. \& D.M. TEIXEIRA. 1984. Um estudo comparativo preliminar sobre as Avifaunas das restingas do leste do Brasil, p.3434-349. In: L.D. LACERDA; D.S.D. ARAúJo; R. CERQUeIRA \& B. TURCQ (Eds). Restingas: origem, estrutura e processos. Niterói, Universidade Federal Fluminense, 400p.

REBOUÇAS-SPIEKER, R. 1974. Distribution and differentiation of animals along the coast and in continental island of the state of São Paulo, Brasil. 2. Lizards of the genus Mabuya (Sauria, Scincidae). Pap. Av. Dept. Zool., São Paulo, 28 (12): 197-240.

RizzINI, C.T. 1979. Tratado de Fitogeografia do Brasil. Aspectos sociológicos e florísticos. São Paulo, HUCITEC, vol. 2, 374p.

RocHA, C.F.D. DA. 1988.Ritmo de atividade e microclimatologia do habitat de Liolaemus lutzae (Sauria: Iguanidae). An. Sem. Reg. Ecol., São Carlos, 6: 
269-281.

1990.Reproductive effort in the Brasilian sand lizard Liolaemus lutzae (Sauria Iguanidae). Ciência e Cultura 42 (12): 1203-1206.

1993.The set of defence mechanisms in a tropical sand lizard (Liolaemus lutzae) of southeastern Brazil. Ciência e Cultura 45 (2): 116-122.

Rodrigues, M.T. 1986. Uma nova espécie de Phyllopezus de Cabaceiras: Paraíba:

Brasil; com comentários sobre a fauna de lagartos da área (Sauria, Gekkonidae).

Pap. Av. Dept. Zool., São Paulo, 36 (20): 237-250.

1987. Sistemática, Ecologia e Zoogeografia dos Tropidurus do grupo

torquatus ao sul do Rio Amazonas (Sauria, Iguanidae). Arq. Zool., São Paulo,

31 (3): 105-230.

SCHOENER, T.W. 1968. The Anolis lizards of Binimi: resource partitioning in a complex fauna. Ecology 49 (4): 704-726.

27-39.

SCHMIDT, K.P. 1936. Notes on Brasilian Amphisbaenians. Herpetologica 1: 28-32.

SECPLAN/RN. 1981. Parque Estadual das dunas do Natal. Levantamento de

informações para o plano de manejo. Natal, Fund. Inst. Desenvolv. Rio

Grande do Norte, 61p.

SMITH, H.M. 1941. Snakes, frogs and bromelias. Chicago Nat. 4: 35-43.

SouZA, E.C. DE. 1945. Restinga. Revta bras. Geogr. 7 (4): 662-665.

STEVAUX, M.N. 1993.Estratégia reprodutiva de Mabuya sp (Sauria: Scincidae): um

padrão geral de reprodução para o gênero na Região Neotropical. Revta

Nordestina Biol. 8 (1): 61-86.

TAVARES DE MELO, S. 1983. L'organization des paysages dans la portion nord-orientale de la Paraiba et du Rio Grande do Norte (Bresil). Tese de doutorado, não publicada, Univ. de Bordeaux, 161p.

Tavares, S. 1960. Estudos geobotânicos do Rio Grande do Norte. Arq. Inst. Pesq. agron. Pernambuco 5: 39-51.

VAllejo, L.R. \& M.S. VAllejo. 1981. Contribuição ao estudo dos microartrópodos do "litter" na restinga de Maricá-RJ. Rev. Brasil. Biol. 41 (3): 535-543.

VANZOLINI, P.E. 1953. Sobre a diferenciação geográfica de Gymnodactylus geckoides (Sauria, Gekkonidae). Pap. Av. Dept. Zool., São Paulo, 11 (14): 225-262.

1957. O gênero Coleodactylus (Sauria, Gekkonidae). Pap. Av. Dept. Zool., São Paulo, 13 (1): 1-17.

17: $1-84$.

1968b. Geography of the South American Gekkonidae (Sauria). Pap. Av.

Dept. Zool., São Paulo, 17 (2): 85-112.

1970. Zoologia Sistemática, geografia e a origem das espécies. São

Paulo, Universidade de São Paulo, Série Teses e Monografias IG,3, 56p.

1972. Miscellaneous notes on the ecology of some brasilian lizards 
(Sauria). Pap. Av. Dept. Zool., São Paulo, 26 (8): 83-115.

1974. Ecological and geographical distribution of lizards in Pernambuco, northeastern Brasil (Sauria). Pap. Av. Dept. Zool., São Paulo, 28 (4): 61-90.

1976a. On the lizards of a cerrado-caatinga contact: evolutionary and zoogeographical implications (Sauria). Pap. Av. Dept. Zool., São Paulo, 29 (16): 111-119.

1976b. Two notes on Anotosaura (Sauria, Teiidae). Pap. Av. Dept. Zool., São Paluo, 30 (8): 119-122.

1978. On South American Hemidactylus (Sauria, Gekkonidae). Pap. Av. Dept. Zool., São Paulo, 31 (20): 307-343.

- 1980. Coleodactylus septentrionalis, sp. n.,with notes on the distribution of the genus (Sauria, Gekkonidae). Pap. Av. Dept. Zool., São Paulo, 34 (1): $1-9$.

1981. A quasi-historical approach to the natural history of the differentiation of reptiles in tropical geographic isolates. Pap. Av. Dept. Zool., São Paulo, 34 (19): 189-204.

1982. A new Gymnodactylus from Minas Gerais, Brazil, with remarks on the genus, on the area and on montane endemism in Brazil. Pap. Av. Dept. Zool., São Paulo, 34 (29): 403-413.

VANZOLINI, P.E. \& A.M.M. RAMOS. 1977. A new species of Colobodactylus, with notes on the distribution of a group of stranded microteiid lizards (Sauria, Teiidae). Pap. Av. Dept. Zool., São Paulo, 31 (3): 19-47.

VAnZOLINI, P.E. \& N. Gomes. 1979. On Tropidurus hygomi: redescription, ecological notes, distribution and history (Sauria, Iguanidae) Pap. Av. Dept. Zool., São Paulo, 32 (21): 243-259.

Vanzolini, P.E.; A.M.M. Ramos-Costa \& L.J. VitT. 1980. Répteis das Caatingas. Rio de Janeiro, Academia Brasileira de Ciências, VI+161p. 